\title{
Article
}

\section{Mothers' perceptions of family centred care in neonatal intensive care units}

Finlayson, Kenneth William, Dixon, Annie, Smith, Chris, Dykes, Fiona Clare and Flacking, Renee

Available at http://clok.uclan.ac.uk/11258/

Finlayson, Kenneth William ORCID: 0000-0002-1287-7630, Dixon, Annie, Smith, Chris, Dykes, Fiona Clare ORCID: 0000-0002-2728-7967 and Flacking, Renee ORCID: 0000-0002-4013-1553 (2014) Mothers' perceptions of family centred care in neonatal intensive care units. Sexual \& Reproductive Healthcare, 5 (3). pp. 119-124. ISSN 18775756

It is advisable to refer to the publisher's version if you intend to cite from the work. http://dx.doi.org/10.1016/j.srhc.2014.06.003

For more information about UCLan's research in this area go to http://www.uclan.ac.uk/researchgroups/ and search for < name of research Group>.

For information about Research generally at UCLan please go to http://www.uclan.ac.uk/research/

All outputs in CLoK are protected by Intellectual Property Rights law, including Copyright law. Copyright, IPR and Moral Rights for the works on this site are retained by the individual authors and/or other copyright owners. Terms and conditions for use of this material are defined in the policies page.

\section{CLoK}

Central Lancashire online Knowledge www.clok.uclan.ac.uk

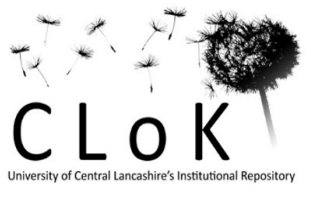




\section{AUTHOR QUERY FORM}

\begin{tabular}{|l|l|l|}
\hline & Journal: SRHC & $\begin{array}{l}\text { Please e-mail or fax your responses and any } \\
\text { corrections to: }\end{array}$ \\
$\begin{array}{l}\text { E-mail: corrections.eseo@elsevier.toppanbestset.com } \\
\text { ELSEVIER }\end{array}$ & Article Number: 143 & \begin{tabular}{l} 
Fax: +852 2897 5170 \\
\hline
\end{tabular} \\
\hline
\end{tabular}

Dear Author,

Please check your proof carefully and mark all corrections at the appropriate place in the proof (e.g., by using on-screen annotation in the PDF file) or compile them in a separate list. To ensure fast publication of your paper please return your corrections within 48 hours.

For correction or revision of any artwork, please consult http://www.elsevier.com/artworkinstructions.

We were unable to process your file(s) fully electronically and have proceeded by
Scanning (parts of) your article
Rekeying (parts of) your article
Scanning the artwork

Any queries or remarks that have arisen during the processing of your manuscript are listed below and highlighted by flags in the proof. Click on the ' $Q$ ' link to go to the location in the proof.

\begin{tabular}{|c|c|c|}
\hline $\begin{array}{l}\text { Location in } \\
\text { article }\end{array}$ & \multicolumn{2}{|c|}{$\begin{array}{l}\text { Query / Remark: click on the Q link to go } \\
\text { Please insert your reply or correction at the corresponding line in the proof }\end{array}$} \\
\hline Q1 & \multicolumn{2}{|c|}{$\begin{array}{l}\text { Please confirm that given names and surnames have been identified correctly and are } \\
\text { presented in the desired order. }\end{array}$} \\
\hline Q2 & \multicolumn{2}{|l|}{ Please check this website address and confirm that it is correct. } \\
\hline Q3 & \multicolumn{2}{|l|}{ Please supply the year of publication for Reference 2 . } \\
\hline Q4 & \multicolumn{2}{|l|}{ Please supply author name for Reference 40 . } \\
\hline & $\begin{array}{l}\text { Please check this box or indicate your approval if } \\
\text { you have no corrections to make to the PDF file }\end{array}$ & \\
\hline
\end{tabular}


Highlights

- Family Centred Care is the model of healthcare adopted by UK neonatal units. 12 mothers were interviewed about their experiences of Family Centred Care. - Mothers found it difficult to take on their maternal role in these environments. • Mothers struggled to form authentic, trusting relationships with neonatal staff. $\bullet$ Key components of Family Centred Care were not recognized by these mothers.

Please cite this article in press as: Kenneth Finlayson, Annie Dixon, Chris Smith, Fiona Dykes, Renee Flacking, Mothers' perceptions of family centred care in neonatal intensive care units, Sexual \& Reproductive Healthcare (2014), doi: 10.1016/j.srhc.2014.06.003 


\title{
Mothers'perceptions of family centred care in neonatal intensive care units
}

\author{
at Kenneth Finlayson a,*, Annie Dixon ${ }^{\mathrm{b}}$, Chris Smith ${ }^{\mathrm{c}}$, Fiona Dykes ${ }^{\mathrm{c}}$, Renee Flacking ${ }^{\mathrm{c}, \mathrm{d}}$ \\ a Research in Childbirth and Health Unit (ReaCH), School of Health, University of Central Lancashire, Preston, UK \\ b Organisational Learning \& Development, Pennine Care NHS Foundation Trust, Greater Manchester, UK \\ ${ }^{c}$ Maternal and Infant Nutrition and Nurture Unit (MAINN), School of Health, University of Central Lancashire, Preston, UK \\ d School of Health and Social Studies, Dalarna University, Falun, Sweden
}

\section{A R T I C L E I N F O}

\section{Article history:}

Received 17 October 2013

Received in revised form 19 May 2014

Accepted 4 June 2014

\section{Keywords:}

Family centred care

Infant

Mother

Neonatal intensive care units

Preterm birth

Qualitative research

\begin{abstract}
A B S T R A C T
Objective: To explore mothers' perceptions of family centred care (FCC) in neonatal intensive care units (NICUs) in England.

Design: The qualitative experiences of 12 mothers from three NICUs in the UK were elicited using individual interviews. A thematic network analysis was conducted on the transcribed interviews Main outcome measures: A central global theme supported by a number of organizing themes were developed reflecting the views of the mothers and their experiences of FCC.

Results: A global theme of "Finding My Place" was identified, supported by six organizing themes: Mothering in Limbo; Deference to the Experts; Anxious Surveillance; Muted Relations, Power Struggles and Consistently Inconsistent. Mothers experienced a state of liminality and were acutely sensitive to power struggles, awkward relationships and inconsistencies in care. To try to maintain their equilibrium and protect their baby they formed deferential relationships with health professionals and remained in a state of anxious surveillance.

Conclusions: This study illustrates that despite the rhetoric around the practice of FCC in NICUs, there was little in the mother's narratives to support this. It is of the utmost importance to minimize the consequences of the liminal experience, to improve staff-_mother interactions and to facilitate mothers' opportunities to be primary caregivers.
\end{abstract}

(c) 2014 Published by Elsevier B.V.

\section{Introduction}

The term "family centred care" (FCC) has been used to describe a model of health care which recognizes the significant role played by family members in the well-being of a hospitalized child or infant [1]. Although there is no simple definition for FCC, the Institute of Patient- and Family-Centered Care [2] describes it as "an approach to the planning, delivery, and evaluation of health care that is grounded in mutually beneficial partnerships among health care providers, patients, and families". This rather broad definition reflects the multi-faceted nature of FCC and its application in different contexts and different cultures. Within neonatology, FCC has evolved into an extensive spectrum of services and activities incorporating a range of environmental, educational, psycho-social and behavioral characteristics all of which seek to place the family at the heart of care planning [3-5]. In the last two decades, FCC based interventions have been developed to support the parent-child attachment relationship [6-9]. There is convincing evidence about the

\footnotetext{
* Corresponding author. Tel.: +4401772 893824 .

E-mail address: kwfinlayson1@uclan.ac.uk (K. Finlayson).
}

positive effects of Kangaroo Mother Care [9-11], but studies suggest there are huge differences in the provision of facilities to enable skinto-skin care and parental involvement [12,13]. A recent European survey, for example, found that $100 \%$ of included units in Sweden had reclining chairs near babies' cots and 100\% had beds for the parents whilst in the UK these figures were $11 \%$ and $77 \%$ respectively [14].

The comprehensive nature of FCC is perhaps best illustrated by Harrison's "Principles for Family Centered Neonatal Care" [15]. This document, drawn up by a committee including neonatologists, neonatal nurses and the parents of premature infants, highlights 10 key strategies most of which revolve around the formation of authentic, collaborative parent-health professional relationships in a supportive, family-friendly environment (Table 1 ).

Collaboration between nurses and parents is essential for implementing FCC in NICUs [16]. A positive and trustful staff--mother relationship enables mothers to connect to their infants whereas a negative relationship may lead to disconnection [17,18]. Furthermore, when mothers feel emotionally supported and have trust in their relationships with nurses, the transition from traumatized onlookers to competent, confident parents is greatly enhanced $[19,20]$. Reis, Remple, Scott, Brady-Fryer and Van Aerde [21] suggest that 
Table 1

A summary of "The Principles for Family-Centred Neonatal Care" [15].

1. FCC in neonatal care should be based on open and honest communication between parents and professionals.

2. Parents should be able to work with professionals in making informed choices based on access to the same facts and uncertainties as those available to professionals.

3. In situations involving high morbidity/mortality and/or medical controversy fully informed parents should have the right to make decisions regarding aggressive treatment for their infants.

4. Expectant parents should be given information about adverse pregnancy outcomes and have the opportunity to state treatment preferences in the event of a premature delivery.

5. Parents and professionals must work together to acknowledge and alleviate the pain of infants.

6. Parents and professionals must work together to ensure an appropriate environment is maintained.

7. Parents and professionals must work together to ensure the safety and efficacy of medical treatments.

8. Parents and professionals must work together to promote parenting skills including active involvement with their hospitalized infant.

9. Parents and professionals must work together to promote long term follow up for NICU survivors.

10. Parents and professionals must acknowledge that treatment should be based on compassion and that over-treatment as well as undertreatment may harm the infant.

the behaviour and attitude of nurses is the most significant factor affecting parent's satisfaction with their NICU experience. Studies also suggest that the neonatal context encourages close relationships between parents and nurses and may even turn the parent-infant dyadic relationship into a "triadic" relationship (i.e. infant-_parent-staff) [22].

In the UK, government initiatives [23] and parental support groups [24] all advocate the implementation of FCC in neonatal settings. Recent attempts to monitor the impact and effectiveness of these policies suggest there is "considerable variation in the familycentredness of the units" in the UK [3]. The POPPY Project [25], a nationwide evaluation of FCC in UK neonatal units, identified gaps in the provision of psycho-social support for parents as well as practical deficiencies in the provision of private spaces and roomingin facilities. The relational aspects of FCC are highlighted in a UK report [26] where the authors found general satisfaction with most aspects of care. However, almost half of the participants felt they did not have as much involvement in discussions about their baby's care as they would have liked and more than a quarter were not offered any form of emotional support while they were on the unit. The insights revealed by large scale, retrospective surveys contribute to an increased understanding of FCC practice but the direct experiences of parents are likely to deepen and enhance this understanding. As far as we are aware no prospective, qualitative studies of FCC in UK NICUs exist. With this in mind we set out to explore mothers', fathers' and neonatal nurses' perceptions of FCC at three NICUs in England. This paper reports on the views of the mothers.

\section{Methods}

\section{Design and setting}

Three NICUs in the North West of England, of differing size and with different populations, were selected to ensure a broad range of views were represented. Two of the units were district based, 2025 cot facilities providing special care, high dependency care and intensive care, and the third, one of the largest in the UK, was a 54 cot regional unit providing all of the above levels of care as well as specialist facilities and expertise for extremely preterm infants. All three units had a family room where parents and siblings could relax and have a cup of tea or coffee and two of the units had facilities (up to three bedrooms within the unit) for parents to stay overnight. All of the units offered 24 hlaccess to parents though in reality visits were informally "discouraged" between 23:00 hl and 07:00 $\mathrm{h}$. The unit manager's at each of the three sites identified Family Centered Care as the model of care practised on the unit.

\section{Procedures}

A convenience sample of potential participants was initially identified by the admissions officer at the units. English speaking mothers over the age of 16 whose baby had been treated on the unit for 7 days or more were considered eligible for inclusion. Mothers whose infants were receiving intensive care at the time of the interviews were excluded as they may have found it intrusive and insensitive. Eligible mothers were given an information sheet and a consent form. Women who agreed to interview were contacted by one of the researchers and a suitable date and venue arranged by mutual consent. All of the interviews were conducted in a private room in the hospital and were recorded using a mini-recording device. All of the interviews took place whilst the mother's baby was being cared for on the unit. The questions were based on Helen Harrison's Principles of Family Centered Neonatal Care [15] (Table 1) and current understandings about the components of FCC. Participants were also given the opportunity to discuss any other issues related to their experience of FCC. The interviews lasted between $45 \mathrm{~min}$ and $2 \mathrm{~h}$ and were conducted by an independent researcher (KF) with no previous experience of spending time on a NICU either as a nurse or a parent. None of the research team worked on any of the three NICUs.during the course of the study.

Ethical approval was obtained by an independent NHS ethics committee and the university ethics committee.

Forty nine mothers were identified as eligible to take part during the 6 month qualitative recruitment phase. Eleven of these left the unit before being approached by the researcher. Thirty eight were asked if they would like to be interviewed and 12 agreed and consented. The 28. women who declined to be interviewed were not asked to give reasons for their decision.

\section{Participants}

The 12. mothers (four from each unit) ranged in age from 21 to 40 years (mean $=26$ ). Eleven out of the 12 identified themselves as "White, British" and the other identified herself as "Asian, British". Six of the mothers were university educated, two had left school at 18 and the remaining four had received no formal education beyond the age of 16 . None of the mothers had spent any time in a NICU prior to this birth. All of the participants had a partner or husband who spent time on the unit and five of the 12 mothers had elder siblings who rarely visited the unit. Most of the mothers spent extended periods of time on the units, ranging from 5 to 70 hLper week (mean $=37 \mathrm{~h}$ ) although none of them stayed overnight with their infants until they were getting ready to leave the unit. [This was primarily because there was no obvious place to sleep or rest near the cot, e.g. a reclining chair or sofa bed]. The infants were all born very preterm ( $<32$ gestational weeks), with an average gestational age of 29 weeks (range $=25-31$ weeks). At birth, the infants weighed between 595-1517g and at the time of the interviews the infants had been receiving neonatal care for an average of 34 days (range $=11-102$ days).

\section{Analysis}

For the data analysis we used thematic networks analysis [27]. Each of the interviews was transcribed by a member of the research team and, through an iterative process involving two other 


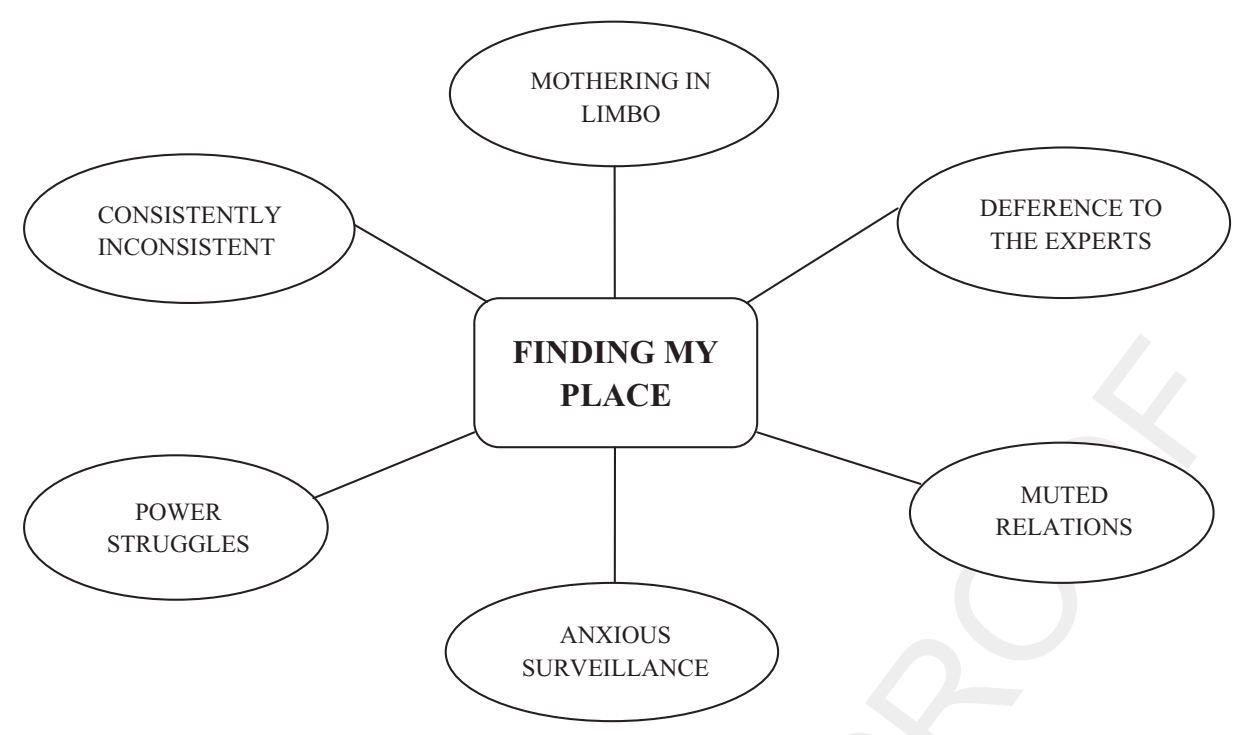

Fig. 1. Global theme and organizing themes.

members of the team (blinded), basic themes were extracted from the data. These basic themes were then discussed by all members of the team to generate organizing themes which were then used to develop a principal organizing metaphor or global theme. All of the mothers names were changed to codes and then to pseudonyms.

\section{Results}

A global theme of Finding My Place was identified within the context of providing FCC as the core model of care in a NICU. All of the mothers in the study described their initial shock at having a premature baby. They found it difficult to "find their place" having become a mother 2-3. months before they were expecting to take on such a role. This was challenging in itself but became even more difficult within the technocratic and overtly clinical environment of a NICU. Thus the global theme utilizes the metaphor of "place" to describe the overarching experience of mothers in this situation and setting. This global theme was underpinned by six organizing themes encapsulated by the following descriptions: Mothering in Limbo; Deference to the Experts; Anxious Surveillance; Muted Relations, Power Struggles and Consistently Inconsistent (see Fig. 1). These themes are described below.

\section{Mothering in limbo}

For all of the participants there was a tangible sense of disappointment at their inability to assume a "normal" maternal role. Trying to come to terms with the shock of having a premature baby, their subsequent concern over the child's well-being and their limited capacity to mother in an unfamiliar environment left many participants feeling alienated from their maternal expectations. One mother expressed this sense of liminality (the sense of disorientation that occurs when pre-motherhood does not resolve into motherhood) as a state of betwixt and between, in terms of not being able to take her baby home to the place where she assumed she would be able to provide a nurturing maternal environment.

At the end of the day I'm a mother but I don't feel very much like a mum. It hasn't sunk in because the baby isn't home and doesn't rely on me.,(Laura)
Similarly, another mother talked about the surreal nature of having a welcome home prepared for her baby's arrival and the subsequent disappointment of not having a baby to take there

It was just strange, especially at first . . there were all sorts of congratulations cards and balloons and gifts.... it was all round the house but there was no baby there. (Julie)

In accord with the philosophy of FCC all of the units offered unrestricted access to parents though this did not necessarily translate into mothers developing a bond with their child or establishing any sense of active involvement or ownership. This was particularly apparent when babies were first admitted or placed in an incubator, but even after several weeks when mums. were preparing to leave the NICU with their infant, some of them were still struggling to come to terms with their maternal role.

Today is the first time I've been on my own with her and it's bad in a way because it should be really nice but it's not really. Although it's great that I'm on my own with her, it's just in this hospital room. . . . it just feels like pretend still . . like I pretended to have a baby. . . that's what it feels like. (Julie)

\section{Deference to the experts}

In general, mothers were extremely grateful to the health professionals looking after their infants. Many of them acknowledged that they were in no position to collaborate effectively during the first few days of admission, particularly when their babies were in intensive care, and actively devolved their decision making to the professionals.

To be honest we had a baby three months early and she's tiny and in an incubator so you do whatever the doctors say at first because they're the experts. (Rachel)

By handing over medical decisions and, more importantly, care responsibilities to health professionals on arrival, mothers found it difficult to redress the balance of power as their time on the unit progressed. Some mothers felt disenfranchised and impotent in their relations with health professionals and this affected their ability to form meaningful bonds with their babies. One mother highlighted the submissive nature of her relationship with the nursing staff by describing the moment when she first held her baby. 
I asked the nurse if it was ok to have a cuddle and she said, "you should have been having at least one a day", but at the start I didn't realise you could ask them. (Laura)

\section{Anxious surveillance}

Most of the mothers spent hours in the neonatal unit sitting beside their baby's cot or incubator. These protracted vigils gave them the opportunity to observe any obvious or subtle changes in their baby's well being. Mothers often struggled to come to terms with the contrast of being an attentive observer within the rather harsh, machinery laden, clinical environment. The constant beeps, buzzes and flashing lights of the medical equipment often added to their anxiety, especially if it hadn't been properly explained to them

When the red light is flashing and that alarms going off I'm having palpitations wondering why nobody was doing anything. (Mary)

As they adapted to the overtly technocratic culture of the units and the monitoring routines of the staff, mothers became more familiar with the technology and adjusted their behavior accordingly - sometimes placing more emphasis on the machines rather than their baby

You're sitting there and I feel for most of the time I'm looking at the monitors more than I'm looking at xxx [the baby]. (Ruth)

As well as attentively watching their baby and gazing at the monitors, mothers also described their surveillance of health professionals, especially nurses. They watched nurses subtly and carefully, constantly evaluating and re-evaluating each nurses' ability to offer high quality care - a combination of affection and professional expertise. When members of staff were not perceived to offer safe, high quality care this gave rise to concern and anxiety. One mother stated

It worries me and my partner this last week as we've noticed the different care you can get with nurses and the difference in attentiveness . . . we sometimes worry who's looking after him. (Laura)

\section{Muted relations}

Some mothers found it difficult to have open and honest communications with the staff. Feelings of frustration, disappointment or anger were often suppressed to avoid potentially damaging confrontations. This contributed to some mothers only partially engaging with the nursing staff feeling that any kind of strong emotional expression might reflect badly on them as mothers and, potentially, affect the care of their baby.

I wouldn't talk to them [nurses] about feeling upset or depressed or if I had worries about her or anything, in case they thought I was some kind of psychotic mother. I've never felt. . . I just couldn't speak to them about anything personal or anything like that. (Jane)

Sometimes their frustrations related to an aspect of care which mothers felt had been overlooked or neglected, e.g. arriving on the unit to find their baby crying and alone, whilst at other times mothers found that care had been given without their consent or permission. One mother described her feelings

I was mortified when someone gave my child their first bath. I felt like saying, 'you shouldn't have done that . . . that's my baby. (Mary)

This could be chronically distressing for some mothers particularly when their frustrations related to the behaviour of the same health professional or professionals. One mother described her persistent efforts to establish relationships with two nurses who regularly dismissed her concerns or failed to answer her queries.

And I dread those two nurses being with $\mathrm{xxx}$ [the baby]. I just think I'm gonna have a nightmare day today. If I want to ask anything then I feel like I can't and it has got to the point, with one of the nurses, where I feel like I just can't ask any questions. So I spend the whole day standing there worrying about it. (Sarah)

\section{Power struggles}

As stated, most of the mothers initially devolved treatment and care responsibilities to the health professionals. More often than not, this initial deference created a submissive relationship with health professionals which proved difficult to rectify during their time on the unit. This subtle power imbalance became the medium through which the relational aspects of FCC were practised and negotiated. Some mothers chose to maintain their submissive role while others sought to establish their maternal identity by actively seeking information about their baby and confronting staff if their views were ignored.

... I'm relentless at saying things I believe in. I sit there and watch him every day and I know what's right and what's not right with him. I'm not medically trained but I know when he's alright and when he's not alright. He has different nurses every time and they don't see him every day. . . he doesn't have the same nurse watching him every day. (Rachel)

However, addressing the balance of power often proved difficult and the desire to claim a sense of maternal responsibility occasionally led to struggle rather than collaboration. One mother, when recounting a conversation about tube feeding, described the encounter in the following manner.

And they said, "you don't need to concern yourself with that, we need to concern ourselves with that". And I was like, actually I don't agree, I think I need to know that as well cos I'm his mum. (Sarah)

Another mother, having spent an average of 7 hla day on the unit over a 5 week period, described how her growing maternal confidence and familiarity with the units' routines and procedures gave her the courage to challenge previously accepted decisions and behaviours

I know my daughter better than anyone on here. . . I won't be fobbed off. I'll ask questions and if I'm not happy with it I'll tell the person I'm not happy with it. (Ruth)

\section{Consistently inconsistent}

When reflecting on their experiences of being in a NICU and their overall perceptions of FCC, all of the mothers highlighted staff inconsistencies as being one of the most upsetting aspects of the care they received. Criticism from nurses about the way they were mothering or being given conflicting pieces of advice or information made them feel incompetent, naïve and disrespected. When asked what improvements could be made to the care she received in a NICU one of the mothers replied:

To me it's just about consistency and every nurse does things differently. One nurse will tell you to do one thing and the next nurse will come in and criticise you cos they wind or feed a baby differently and it makes you feel like crap. (Hannah)

Some mothers related the inconsistency of care to the age of a nurse, their level of experience or knowledge of neonatal care. One mother, when asked to give an example of inconsistency, got visibly distressed as she recounted her attempts to start kangaroo care.

I knew about kangaroo care but I wasn't told about it . . it wasn't explained to me. And I felt stupid asking the nurses, and some nurses were quite helpful and other nurses basically told me a straightforward, "no, she's better off in her cot or an incubator". . . . and that upset me. (Jane) 


\section{Discussion}

Our findings indicate that these mothers were struggling to find their place - thrown into a situation where they had become biological mothers but were not feeling like a mother or acknowledged as "being the mother". They were in a state of liminality, an unsettling period of transition between one social state or status and another $[28,29]$. This phenomenon has been recognized in other NICU contexts and has variously been described as "putting life on hold" [18], being "disenfranchised" [19] or feeling "alienated" [30]. Watson [29] asserts that crisis, uncertainty and powerlessness make parents of very preterm infants into "liminal people". The liminal mothers in our study were acutely sensitive to power struggles, muted relationships and inconsistencies in care and information. To try to maintain their equilibrium and protect their baby they showed deference to the "experts" and remained in a state of anxious surveillance. Similar findings have been reported by others $[18,19,31,32]$ but not in NICUs explicitly identified as using FCC as the primary model of care.

One of the most important aspects to consider in FCC culture is the position of staff in relation to the parents and their perceived expectations of involvement [16,33,34]. Inadequate staffparent interaction and communication can exacerbate parents' sense of isolation from their premature infants $[18,19]$. A change in staff attitude and behaviour from considering parents as "visitors" to being the primary caregivers demands a shift of power and role negotiation, which needs active encouragement by all health professionals [1]. For a shift of power to happen staff-_parent communication is of crucial importance [35]. An individualized approach to care and appropriate communication with parents has been reported to facilitate a positive parent-infant relationship [7]. Appropriate communication could be described as the staff having an authentic presence and a facilitative approach in their way of giving support [36]. Furthermore, although trustful staff-pparent communication may be vital for the development of maternal identity, one also needs to consider how to actively involve parents in order to genuinely practise FCC. Strategies and interventions to increase parental involvement in NICUs have been performed and reported on in different areas. For example, parents have been involved in pain management by holding their preterm infant [37] or encouraged to provide skin-to-skin contact [38]. Physical and emotional closeness are crucial to the physical, emotional and social wellbeing of both the infant and the parent and for the development of a secure bond [39]. Efforts should therefore be made to ensure that parents can remain in the NICU for as long as possible (including overnight stays) in accord with the UN Convention on the Rights of the Child [40]. By adhering to this Convention, Kangaroo Mother Care could be facilitated in all types of settings [9,11]. In our study, parents had access to their infants 24 hLa day, but to minimize the feeling of liminality and to enhance parental involvement, parents need to feel that there is a space and place for them where they can bond with their infant without feeling supervised [5,39,41].

Given the relatively small number of participants from a limited number of sites it is difficult to make generalizations from this study but the interviews were extensive and in-depth and enabled the development of illuminating themes. Unfortunately, we did not have the resources to include non-English speakers in the interviews and, given the significance of communication in FCC, these participants may have yielded additional insights. As discussed above, our themes support findings from similar studies in different geographical locations and this does raise concerns about the general application of FCC in NICU environments. It is also important to stress that the findings from this article may be further contextualized once the interviews with nurses and fathers have been analysed. Previous studies with neonatal nurses have found that their ability to practise the relational aspects of FCC may be compromised by or- ganizational and resource constraints including staff shortages and a lack of training [42]. These factors may limit a nurse's confidence in their ability to practiselFCC as well as the amount of time they are able to spend with parents.

\section{Conclusions}

The aim of this study was to explore mothers' perceptions of FCC in the UK. Our findings indicate that mothers struggled to "find their place" within the highly medicalized NICU setting. Because of the unexpected arrival of their baby and the unfamiliar nature of the NICU environment mothers found it difficult to take up their anticipated maternal role and entered a state of limbo or liminality. This left them feeling vulnerable and exposed to power struggles or muted relationships with nursing staff and sensitive to inconsistencies in care delivery and information exchange. To try to maintain their equilibrium and protect their baby they showed deference to the "experts" and remained in a state of anxious surveillance. These findings illustrate that despite the rhetoric about the practice of FCC as the principle model of care in these neonatal units, there was little in the mother's narratives to support this. Thus, it is of the utmost importance to minimize the potentially disturbing consequences of the liminal experience, to improve staffmother interactions, and to facilitate the opportunities for mothers' to be the primary caregivers. More research is needed in order to understand the barriers to providing FCC from an organizationall and staff perspective.

\section{References}

[1] Moore KA, Coker K, DuBuisson AB, Swett B, Edwards WH. Implementing potentially better practices for improving family-centered care in neonatal intensive care units: successes and challenges. Pediatrics 2003;111(4):450-60.

[2] Institute for Family-Centered Care. http://www.ipfcc.org/pdf/CoreConcepts.pdf] Last accessed 16th April 2014.

[3] Redshaw ME, Hamilton KE. Family centred care? Facilities, information and support for parents in UK neonatal units. Arch Dis Child Fetal Neonatal Ed 2010;95(5):F365-8.

[4] Gooding JS, Cooper LG, Blaine AI, Franck LS, Howse JL, Berns SD. Family support and family-centered care in the neonatal intensive care unit: origins, advances, impact. Semin Perinatol 2011;35(1):20-8.

[5] Heinemann AB, Hellstrom-Westas L, Hedberg Nyqvist K. Factors affecting parents' presence with their extremely preterm infants in a neonatal intensive care room. Acta Paediatr 2013;102(7):695-702.

[6] Als $\mathrm{H}$, Gilkerson L. The role of relationship-based developmentally supportive newborn intensive care in strengthening outcome of preterm infants. Semin Perinatol 1997;21(3):178-89.

[7] Kleberg A, Hellstrom-Westas L, Widstrom AM. Mothers' perception of Newborn Individualized Developmental Care and Assessment Program (NIDCAP) as compared to conventional care. Early Hum Dev 2007;83(6):403-11.

[8] Conde-Agudelo A, Diaz-Rossello JL, Belizan JM. Kangaroo mother care to reduce morbidity and mortality in low birthweight infants. Cochrane Database Syst Rev 2003;(2):CD002771.

[9] Nyqvist KH, Anderson GC, Bergman N, Cattaneo A, Charpak N, Davanzo R, et al. State of the art and recommendations. Kangaroo mother care: application in a high-tech environment. Acta Paediatr 2010;99(6):812-19.

[10] Anderson GC, Chiu SH, Dombrowski MA, Swinth JY, Albert JM, Wada N. Mother-newborn contact in a randomized trial of kangaroo (skin-to-skin) care. J Obstet Gynecol Neonatal Nurs 2003;32(5):604-11.

[11] Nyqvist KH, Anderson GC, Bergman N, Cattaneo A, Charpak N, Davanzo R, et al. Towards universal Kangaroo Mother Care: recommendations and report from the First European conference and Seventh International Workshop on Kangaroo Mother Care. Acta Paediatr 2010;99(6):820-6.

[12] Hamilton K, Redshaw M. Developmental care in the UK: a developing initiative. Acta Paediatr 2009;98(11):1738-43.

[13] Olsson E, Andersen RD, Axelin A, Jonsdottir RB, Maastrup R, Eriksson M. Skin-to-skin care in neonatal intensive care units in the Nordic countries: a survey of attitudes and practices. Acta Paediatr 2012;101(11):1140-6.

[14] Greisen G, Mirante N, Haumont D, Pierrat V, Pallás-Alonso C, Warren I, et al. Parents, siblings and grandparents in the Neonatal Intensive Care Unit. A survey of policies in eight European countries. Acta Paediatr 2009;98(11):1744-50.

[15] Harrison $H$. The principles for family-centered neonatal care. Pediatrics 1993:92(5):643-50.

[16] Saunders RP, Abraham MR, Crosby MJ, Thomas K, Edwards WH. Evaluation and development of potentially better practices for improving family-centered care in neonatal intensive care units. Pediatrics 2003;111(4 Pt 2):e437-49. 
[17] Lupton D, Fenwick J. 'They've forgotten that I'm the mum': constructing and practising motherhood in special care nurseries. Soc Sci Med 2001;53(8):101121.

[18] Flacking R, Ewald U, Nyqvist KH, Starrin B. Trustful bonds: a key to "becoming a mother" and to reciprocal breastfeeding. Stories of mothers of very preterm infants at a neonatal unit. Soc Sci Med 2006;62(1):70-80.

[19] Fenwick J, Barclay L, Schmied V. Struggling to mother: a consequence of inhibitive nursing interactions in the neonatal nursery. J Perinat Neonatal Nurs 2001;15(2):49-64.

[20] Cooper LG, Gooding JS, Gallagher J, Sternesky L, Ledsky R, Berns SD. Impact of a family-centered care initiative on NICU care, staff and families. J Perinatol 2007;27(Suppl. 2):S32-7.

[21] Reis MD, Rempel GR, Scott SD, Brady-Fryer BA, Van Aerde J. Developing nurse/parent relationships in the NICU through negotiated partnership. J Obstet Gynecol Neonatal Nurs 2010;39(6):675-83.

[22] Bialoskurski M, Cox CL, Hayes JA. The nature of attachment in a neonatal intensive care unit. J Perinat Neonatal Nurs 1999;13(1):66-77.

[23] NHS \& Department of Health. Toolkit for high quality neonatal services. 2009.

[24] BLISS. The Bliss baby charter standards. London: Bliss Publication; 2009. p. 1-24.

25] Staniszewska S, Brett J, Redshaw M, Hamilton K, Newburn M, Jones N, et al. The POPPY Study: developing a model of family-centred care for neonatal units. Worldviews Evid Based Nurs 2012;9(4):243-55.

[26] Howell E, Graham C. Parents' experiences of neonatal care - a report on the findings from a national survey. Oxford, UK: 2011.

[27] Attride-Stirling J. Thematic networks: an analytic tool for qualitative research. Qual Res 2001;1(3):385-405.

[28] Van Gennep A. The rites of passage. In: Davis-Floyd R, editor. Birth as an American rite of passage. Chicago, IL: University of Chicago Press; 1966.

[29] Watson G. Parental liminality: a way of understanding the early experiences of parents who have a very preterm infant. J Clin Nurs 2011;20(9-10):1462-71.
[30] Jackson K, Ternestedt BM, Schollin J. From alienation to familiarity: experiences of mothers and fathers of preterm infants. J Adv Nurs 2003;43(2):120-9.

[31] Wigert H, Johansson R, Berg M, Hellstrom AL. Mothers' experiences of having their newborn child in a neonatal intensive care unit. Scand J Caring Sci 2006;20(1):35-41.

[32] Flacking R, Ewald U, Starrin B. "I wanted to do a good job": experiences of becoming a mother and breastfeeding in mothers of very preterm infants after discharge from a neonatal unit. Soc Sci Med 2007;64(12):2405-16.

[33] Davis L, Mohay H, Edwards H. Mothers' involvement in caring for their premature infants: an historical overview. J Adv Nurs 2003;42(6):578-86.

[34] Franck LS, Axelin A. Differences in parents', nurses' and physicians' views of NICU parent support. Acta Paediatr 2013;102(6):590-6.

[35] Lindberg B, Axelsson K, Öhrling K. The birth of premature infants: experiences from the fathers' perspective. J Neonatal Nurs 2007;13(4):142-9.

[36] Schmied V, Beake S, Sheehan A, McCourt C, Dykes F. Women's perceptions and experiences of breastfeeding support: a metasynthesis. Birth 2011;38(1):49-60.

[37] Axelin A, Salantera S, Kirjavainen J, Lehtonen L. Oral glucose and parental holding preferable to opioid in pain management in preterm infants. Clin J Pain 2009;25(2):138-45.

[38] Flacking R, Ewald U, Wallin L. Positive effect of kangaroo mother care on long-term breastfeeding in very preterm infants. J Obstet Gynecol Neonatal Nurs 2011;40(2):190-7.

[39] Flacking R, Lehtonen L, Thomson G, Axelin A, Ahlqvist S, Moran VH, et al. Closeness and separation in neonatal intensive care. Acta Paediatr 2012;101(10):1032-7.

[40] UN convention on the rights of the child. Br Med J 1989;298(6686):1477 \&.

[41] Flacking R, Dykes F. "Being in a womb" or "playing musical chairs": the impact of place and space on infant feeding in NICUs. BMC Pregnancy Childbirth 2013;13(1):179.

[42] Higman W, Shaw K. Nurses' understanding about the delivery of family centered care in the neonatal unit. J Neonatal Nurs 2008;14(6):193-8. 